\title{
A REMARK CONCERNING SUFFICIENCY THEOREMS FOR THE PROBLEM OF BOLZA ${ }^{1}$
}

\author{
E. J. MCSHANE
}

In recent years there have appeared several sufficiency theorems for minima in Bolza problems in which there is no assumption that the curve in question is normal. However, it is assumed in all these theorems that the multiplier rule, the transversality conditions and the strengthened conditions of Weierstrass, Clebsch and Jacobi hold for a set of multipliers with $\lambda_{0}>0$; whereas, among the necessary conditions the multiplier rule and the Weierstrass and Clebsch conditions have been shown ${ }^{2}$ to hold for multipliers with $\lambda_{0} \geqq 0$.

In the present note we close this slight gap by showing that the sufficiency theorems hold if we assume $\lambda_{0}$ non-negative instead of positive. The only case needing discussion is $\lambda_{0}=0$. In this case we show that the curve satisfying the hypothesis is isolated; no neighboring curve satisfies the side-equations and end-conditions. The curve is thus a minimizing curve in a trivial sense. Less trivially, we show $^{3}$ that the curve is a normal proper minimizing curve for a Mayer problem in which the end-conditions consist of a subset of the original end-conditions and the function to be minimized is a linear combination of the original end-functions.

The problem is that of minimizing a functional

$$
J[y]=g\left(x_{1}, y\left(x_{1}\right), x_{2}, y\left(x_{2}\right)\right)+\int_{x_{1}}^{x_{2}} f\left(x, y(x), y^{\prime}(x)\right) d x
$$

in the class of curves $y^{i}=y^{i}(x), x_{1} \leqq x \leqq x_{2}, i=1, \cdots, n$, which satisfy certain differential equations

$$
\phi_{\alpha}\left(x, y(x), y^{\prime}(x)\right)=0, \quad \alpha=1, \cdots, m<n,
$$

and certain end-conditions

$$
\psi_{\mu}\left(x_{1}, y\left(x_{1}\right), x_{2}, y\left(x_{2}\right)\right)=0, \quad \mu=1, \cdots, p \leqq 2 n+2 .
$$

We suppose the usual conditions ${ }^{4}$ of continuity and differentiability

1 Presented to the Society, September 8, 1939.

2 E. J. McShane, On multipliers for Lagrange problems, American Journal of Mathematics, vol. 61 (1939), pp. 809-819.

${ }^{3}$ At the suggestion of Professor M. R. Hestenes.

${ }^{4}$ See, e.g., G. A. Bliss, The problem of Bolza in the calculus of variations, Annals of Mathematics, (2), vol. 33 (1932), pp. 261-273. 
satisfied, as well as the usual conditions on the ranks of the matrices of derivatives of $\phi_{\alpha}$ and $\psi_{\mu}$.

Consider a curve $C: y_{i}=y_{i}(x), x_{1} \leqq x \leqq x_{2}$, which is of class $C^{\prime \prime}$ and satisfies equations (2) and (3). If $\lambda_{0}$ is a constant and $\lambda_{\alpha}(x)$ a set of functions, we define

$$
F\left(x, y, y^{\prime}, \lambda\right)=\lambda_{0} f\left(x, y, y^{\prime}\right)+\lambda_{\alpha}(x) \phi_{\alpha}\left(x, y, y^{\prime}\right) .
$$

The statements that $C$ satisfies the multiplier rule, the strengthened Weierstrass condition $\mathrm{II}_{N}{ }^{\prime}$ and the strengthened Clebsch condition III' shall have their usual meanings. ${ }^{5}$ The curve $C$ satisfies the transversality condition if there are numbers $e_{1}, \cdots, e_{p}$ such that

$$
\left.\left(F-y_{i}^{\prime} F_{y_{i^{\prime}}}\right) d x\right|_{1} ^{2}+\left.F_{y_{i^{\prime}}} d y_{i}\right|_{1} ^{2}+\lambda_{0} d g+e_{\mu} d \psi_{\mu}=0
$$

is an identity in $d x_{1}, d x_{2}, d y_{i 1}$ and $d y_{i 2}$.

For normal problems, in which we can suppose $\lambda_{0}=1$, the second variation of $J[y]$ due to the variations $\left[\xi_{1}, \xi_{2}, \eta(x)\right]$ is given by a functional ${ }^{6}$

$$
Q\left[\xi_{1}, \eta\left(x_{1}\right), \xi_{2}, \eta\left(x_{2}\right)\right]+\int_{x_{1}}^{x_{2}} 2 \omega\left(x, \eta, \eta^{\prime}\right) d x .
$$

Even for abnormal problems we shall say that the curve $C$ satisfies condition IV $^{\prime}$ if the functional (5) is positive for every variation $\left[\xi_{1}, \xi_{2}, \eta(x)\right]$ not identically zero which satisfies the equations of variation

$$
\begin{array}{rlrl}
\phi_{\alpha y_{i}} \eta_{i}+\phi_{\alpha y_{i}} \eta_{i}^{\prime} & \equiv 0, & \alpha=1, \cdots, m, \\
\Psi_{\mu}\left(\xi_{1}, \eta\left(x_{1}\right), \xi_{2}, \eta\left(x_{2}\right)\right) & =0, & \mu & =1, \cdots, p,
\end{array}
$$

where as usual

$$
\begin{aligned}
\Psi_{\mu}\left(\xi_{1}, \eta_{1}, \xi_{2}, \eta_{2}\right)=\left(\psi_{\mu x_{1}}+y_{i}^{\prime}\left(x_{1}\right) \psi_{\mu y_{i 1}}\right) \xi_{1}+\psi_{\mu y_{i 1}} \eta_{i 1} \\
+\left(\psi_{\mu x_{2}}+y_{i}^{\prime}\left(x_{2}\right) \psi_{\mu y_{i 2}}\right) \xi_{2}+\psi_{\mu y_{i 1}} \eta_{i 2} .
\end{aligned}
$$

We do not need the specific form of the quadratic function $Q$ or of $2 \omega$, but for a later paragraph it will be important to note that in (5) the integrand $f$ enters only via the combination $F\left(x, y, y^{\prime}, \lambda\right)$, while in the coefficients of $Q$ the functions $g$ and $\psi_{\mu}$ enter only in the combination $\lambda_{0} g+e_{\mu} \psi_{\mu}$ and its derivatives.

${ }^{5}$ See, e.g., M. R. Hestenes, Sufficient conditions for the problem of Bolza in the calculus of variations, Transactions of this Society, vol. 36 (1934), pp. 795, 809, 813.

${ }^{6}$ For this computation, see, e.g., G. A. Bliss and M. R. Hestenes, Sufficient conditions for a problem of Mayer in the calculus of variations, Transactions of this Society, vol. 35 (1933); in particular, pp. 311, 312. 
The sufficiency theorem referred to in the introduction is the following. ${ }^{7}$

THEOREM 1. If the curve $C$ is of class $C^{\prime \prime}$ and there are multipliers $\lambda_{0}>0, \lambda_{\alpha}(x)$ with which it satisfies the multiplier rule, the transversality condition and the conditions $\mathrm{II}_{N}{ }^{\prime}, \mathrm{III}^{\prime}$ and $\mathrm{IV}^{\prime}$, then there exists a neighborhood $\mathcal{F}$ of $C$ in $(x, y)$-space and a neighborhood $N$ of $\left(x_{1}, y\left(x_{1}\right), x_{2}, y\left(x_{2}\right)\right)$ in $\left(x_{1}, y_{1}, x_{2}, y_{2}\right)$-space with the following properties. If $\bar{C}: y=\bar{y}(x)$, $\bar{x}_{1} \leqq x \leqq \bar{x}_{2}$, lies in $\mathcal{F}$, and the point $\left(\bar{x}_{1}, \bar{y}\left(\bar{x}_{1}\right), \bar{x}_{2}, \bar{y}\left(\bar{x}_{2}\right)\right)$ is in $N$, and $\bar{C}$ satisfies conditions (2) and (3), then either $\bar{C}$ is identical with $C$ or $J[\bar{y}]>J[y]$.

We now establish a slight generalization of this theorem.

TheOREM 2. In Theorem 1, the hypothesis $\lambda_{0}>0$ can be weakened to $\lambda_{0} \geqq 0$. Moreover, if the other hypotheses of Theorem 1 hold with multipliers $\lambda_{0}=0, \lambda_{\alpha}(x)$, the conclusion can be strengthened by replacing the last clause by the words "then $\bar{C}$ is identical with $C . "$

Observe that $f, g, \lambda_{0}$ never occur in the hypotheses save in the combinations $\lambda_{0} f, \lambda_{0} g$ and their derivatives. Suppose $\lambda_{0}=0$. All hypotheses continue to be satisfied if we change the problem by replacing $\lambda_{0}$ by 1 and replacing $f$ and $g$ by 0 . For this new problem the hypotheses of Theorem 1 hold, and clearly the new functional $J[y]$ is identically 0 . Therefore the conclusion of Theorem 1 holds, but the inequality $J[\bar{y}]>J[y]$ is impossible. This establishes Theorem 2 .

Henceforth we assume that the hypotheses of Theorem 2 hold with $\lambda_{0}=0$. The curve $C$ is then surely not normal; if

$$
\left[\xi_{1}^{j}, \xi_{2}^{j}, \eta^{j}(x)\right], \quad j=1, \cdots, p,
$$

is an aggregate of variations satisfying equation (6), the matrix

$$
\left\|\Psi_{\mu}\left(\xi_{1}^{j}, \eta^{j}\left(x_{1}\right), \xi_{2}^{j}, \eta^{j}\left(x_{2}\right)\right)\right\|, \quad \mu, j=1, \cdots, p,
$$

has rank less than $p$. Let $q<p$ be the greatest rank which the matrix (10) can have, and let the set (9) be so chosen that the matrix has rank $q$. By renumbering if necessary, we can bring it about that the upper left minor, corresponding to $\mu, j=1, \cdots, q$, is nonsingular.

Consider now the Mayer problem of minimizing the function

$$
J^{*}[y]=e_{\mu} \psi_{\mu}\left(x_{1}, y\left(x_{1}\right), x_{2}, y\left(x_{2}\right)\right)
$$

${ }^{7}$ For a proof, and for reference to other papers on the subject, see M. R. Hestenes, $A$ direct sufficiency proof for the problem of Bolza in the calculus of variations, Transactions of this Society, vol. 42 (1937), pp. 141-154. 
(where $e_{1}, \cdots, e_{p}$ are the constants entering in the transversality condition) in the class of arcs satisfying the differential equations (2) and the end-conditions

$$
\psi_{\kappa}\left(x_{1}, y\left(x_{1}\right), x_{2}, y\left(x_{2}\right)\right)=0, \quad \kappa=1, \cdots, q .
$$

We shall prove that for this problem the curve $C$ is normal and gives $J^{*}$ a strong proper relative minimum. That is, the conclusion of Theorem 1 holds if we replace the reference (3) by (12) and change $J$ to $J^{*}$.

The normality of $C$ is evident; for this Mayer problem, the analogue of matrix (10) is the upper left $q$-square minor of (10), which is nonsingular.

Equation (11) is the analogue of (1) with

$$
g^{*}\left(x_{1}, y_{1}, x_{2}, y_{2}\right)=e_{\mu} \psi_{\mu}\left(x_{1}, y_{1}, x_{2}, y_{2}\right), \quad f^{*}\left(x, y, y^{\prime}\right) \equiv 0 .
$$

Define

$$
\lambda_{0}^{*}=1, \quad \lambda_{\alpha}^{*}(x)=\lambda_{\alpha}(x) .
$$

Then

$$
\begin{aligned}
F^{*}\left(x, y, y^{\prime}, \lambda^{*}\right) & =\lambda_{0}^{*} f^{*}+\lambda_{\alpha}^{*}(x) \phi_{\alpha}\left(x, y, y^{\prime}\right) \\
& =\lambda_{\alpha}(x) \phi_{\alpha}\left(x, y, y^{\prime}\right)=F\left(x, y, y^{\prime}, \lambda\right) .
\end{aligned}
$$

Since the multiplier rule and conditions $\mathrm{II}_{N}{ }^{\prime}$ and $\mathrm{III}^{\prime}$ involve only $F$ and its derivatives, they are satisfied for our Mayer problem.

If we choose

$$
e_{\kappa}^{*}=0, \quad \kappa=1, \cdots, q,
$$

we find, recalling (4) and (15),

$$
\begin{aligned}
\left.\left(F^{*}-y_{i}^{\prime} F_{y_{i^{\prime}}}^{*}\right) d x\right|_{1} ^{2} & +\left.F_{y_{i^{\prime}}}^{*} d y_{i}\right|_{1} ^{2}+\lambda_{0}^{*} d g^{*}+e_{\kappa}^{*} d \psi_{\kappa} \\
& =\left.\left(F-y_{i} F_{y_{i^{\prime}}}\right) d x\right|_{1} ^{2}+\left.F_{y_{i^{\prime}}} d y_{i}\right|_{1} ^{2}+\lambda_{0} d g+e_{\mu} d \psi_{j^{\prime}}=0
\end{aligned}
$$

identically in $d x_{1}, d x_{2}, d y_{i 1}$ and $d y_{i 2}$. So the transversality condition is satisfied in our Mayer problem.

From (13), (14) and (16) we have

$$
\lambda_{0}^{*} g^{*}+e_{\kappa}^{*} \psi_{\kappa} \equiv \lambda_{0} g+e_{\mu} \psi_{\mu} .
$$

Now in the light of (17) and (15) the sentence after (8) assures us that the sum (5) is identically equal to its analogue for our Mayer problem. Hence condition IV' is satisfied. All the hypotheses of Theorem 1 have now been verified for our Mayer problem, and it follows that $C$ minimizes $J^{*}[y]$, as stated.

UNIVERSITY OF VIRGINIA 